\title{
NIR emitting GdVO 4 :Nd nanoparticles for bioimaging: the role of the synthetic pathway
}

Tecla Carbonati $^{\mathrm{a}}$, Carolina Cionti ${ }^{\mathrm{a}, \mathrm{c}}$, Ewoud Cosaert ${ }^{\mathrm{b}}$, Ben Nimmegeers ${ }^{\mathrm{b}}$, Daniela Meroni ${ }^{\mathrm{a}, \mathrm{c}, *},{ }^{,}$, Dirk Poelman ${ }^{\mathrm{b}, *}$

${ }^{a}$ Università degli Studi di Milano, Department of Chemistry, via Golgi 19, 2013 b3 Milan, Italy

${ }^{b}$ Ghent University, Department of Solid State Sciences, Ghent, Belgium

${ }^{c}$ Consorzio INSTM, via Giusti 9, 50121 Florence, Italy

*Corresponding authors: daniela.meroni@unimi.it ; dirk.poelman@ugent.be

\begin{abstract}
Nd-doped $\mathrm{GdVO}_{4}\left(\mathrm{GdVO}_{4}: \mathrm{Nd}\right)$ represents a promising candidate among near-infrared emitting nanoparticles (NPs) for time-gated bioimaging owing to its non-damaging, non-heating excitation wavelength provided by cheap lasers. The $\mathrm{GdVO}_{4}: \mathrm{Nd}$ synthesis should guarantee a precise control over NPs dimensions along with optimized luminescence properties. Here, we carried out for the first time a systematic study of $\mathrm{GdVO}_{4}: \mathrm{Nd}$ NPs synthetic routes: a simple co-precipitation was compared to colloidal, reverse micelles and sonochemical methods. The NPs were characterized for their structural, morphological, spectral and luminescence lifetime properties, also with respect to a micrometric reference prepared by solid state reaction. All the prepared NPs showed high crystallinity, with spindle-like morphology and dimensions smaller than $150 \mathrm{~nm}$. The synthetic procedure plays a crucial role on the sample purity and crystallite size, leading to notable differences in emission intensity and luminescence decay. The co-precipitation and colloidal syntheses led to particles with suitable morphology and high emission intensity. The optimal dopant content was found to be close to $2 \%$, in order to avoid concentration quenching effects. Luminescence decay lifetimes of nanosized samples are long enough to counter tissue autofluorescence in time-gated applications.
\end{abstract}

\section{Keywords}

Near-infrared luminescence; multimodal imaging; bioimaging; time-gated detection; neodymium; $\mathrm{Nd}^{3+}$ 


\section{Introduction}

Light-emitting materials are widely applied not only in lighting and communication, but also in medicine and biology, where luminescent probes and sensors are extensively adopted. In this respect, fluorescence is widely exploited for disease screening, fluorescence guided high-precision surgical procedures and for both in vitro and in vivo bioimaging ${ }^{1}$. In the latter application, the absorbance of biological tissues must be considered ${ }^{2}$ : therefore, a suitable phosphor should operate in the so-called biological windows (BWs). BWs are three infrared spectral ranges (BW-I from 700 to $950 \mathrm{~nm}, \mathrm{BW}$-II from 1000 to $1350 \mathrm{~nm}$ and BW-III from 1550 to $1870 \mathrm{~nm}^{3}$ ) in which biological tissues become partially transparent, due to a simultaneous reduction in both the absorption and scattering coefficients ${ }^{4}$. Another issue related to in vivo bioimaging concerns the intrinsic fluorescence of biological tissues, known as autofluorescence ${ }^{5}$. The autofluorescence interferes mainly in BW-II and BW-III, within the wavelength range with optimum sensitivity of the InGaAs detectors. However, autofluorescence intensity slightly drops in BW-II between 1050 and 1150 $\mathrm{nm}^{1}$. Therefore, an ideal phosphor for in vivo bioimaging should emit in this BW-II region and should be excited with a wavelength in BW-I, in correspondence to the emission of broadly available laser diodes.

Persistent luminescent materials are promising materials for in vivo bioimaging applications, since their emission lasts for an appreciable amount of time after the excitation has stopped ${ }^{6,7}$. However, this method is limited by the phosphor's confined energy storage capacity and by the necessity of a long and intense afterglow. In this framework, time-gated measurements carried out adopting NIR absorbing and emitting phosphors ${ }^{8}$ are a promising alternative since they can be re-excited directly in vivo. The detection of the emitted light is classified as "time-gated" as it occurs few microseconds after the excitation pulse, in order to cut off short-living tissues' autofluorescence, with a decay time of the order of $100 \mathrm{~ns}^{9}$. The main advantage of the proposed method compared to persistent phosphors thus is the possibility to keep tracking the particles for a very long time (as long as they remain in the body), well past the time persistent luminescence would have decayed to the point of becoming unmeasurable ${ }^{10}$.

For these reasons, NIR emitting nanoprobes, such as rare earth-doped NPs (RENPs), have driven increasing attention in the last years ${ }^{9,11}$ and Nd-based phosphors are among the most promising candidates ${ }^{12,13}$. All $\mathrm{Nd}^{3+}$-doped materials are characterized by three main emissions in the nearinfrared, the most intense of them lies around $1062 \mathrm{~nm}\left({ }^{4} \mathrm{~F}_{3 / 2} \rightarrow{ }^{4} \mathrm{I}_{11 / 2} \text { transition }\right)^{14}$, i.e. in BW-II's ideal range. The main excitation peak lies around $800 \mathrm{~nm}(\mathrm{BW}-\mathrm{I})^{14}$ and corresponds to the ${ }^{4} \mathrm{I}_{9 / 2} \rightarrow$ ${ }^{4} \mathrm{~F}_{5 / 2}+{ }^{2} \mathrm{H}_{9 / 2}$ electronic transition, which is followed by a non-radiative relaxation to the state ${ }^{4} \mathrm{~F}_{3 / 2}$. Del Rosal et al. compared different host species for $\mathrm{Nd}^{3+}$ doping ${ }^{13}$, finding notable differences in 
the luminescence properties of the materials. Among them, $\mathrm{GdVO}_{4}: \mathrm{Nd}$ stands out ${ }^{15-17}$ because it exhibits an excitation maximum really close to $808 \mathrm{~nm}$, which is a non-damaging wavelength, easily provided by a cost-effective laser diode, at which heating due to water absorption is minimised $^{18}$. By contrast, many other Nd-doped NPs display excitation maxima closer to $790 \mathrm{~nm}$, a wavelength responsible for a cell damage $30 \%$ more serious with respect to a $808 \mathrm{~nm}$ radiation ${ }^{19}$. There are currently no official guidelines for safe skin exposure by pulsed sources ${ }^{20}$, but it is accepted that the main risk from IR-A skin exposure (wavelength range 760-1400 $\mathrm{nm}$ ) is tissue heating $^{21}$. In the latter paper, IR-A mice tissue irradiation of $190 \mathrm{~mW} / \mathrm{cm}^{2}$ during 30 minutes was shown not to have any adverse effects. For short-term (up to 10s) skin exposure in the wavelength range 380-3000 nm, European safety standard EN 62471 ${ }^{22}$ specifies an exposure limit $\mathrm{E}\left(\frac{\mathrm{W}}{\mathrm{m}^{2}}\right)<$ $\Delta \mathrm{t}^{-0.75}$, which comes down to $2 \mathrm{~W} / \mathrm{cm}^{2}$ for a $1 \mathrm{~s}$ exposure. Given that at the intended excitation wavelength of around $808 \mathrm{~nm}$, there is a marked minimum in tissue absorption, the safety limit is probably much higher at this wavelength, but there is currently no published information which could confirm this. In view of the high excitation efficiency of $\mathrm{GdVO}_{4}: \mathrm{Nd}$ at $808 \mathrm{~nm}$ and the low tissue absorption of the emitted light at $1064 \mathrm{~nm}$, we are confident that in vivo excitation could be performed with a low power diode laser well below safety limits.

Furthermore, $\mathrm{GdVO}_{4}: \mathrm{Nd}$ nanoparticles could be adopted as a multimodal imaging material ${ }^{23}$, considering that paramagnetic $\mathrm{Gd}(\mathrm{III})$ chelates are the most representative T1-positive contrast agents for magnetic resonance imaging $(\mathrm{MRI})^{24}$. As a matter of fact, multifunctional nanoparticles can provide more information than probes with a single imaging modality, thus widening their scope of application ${ }^{25}$.

Thanks to its high chemical stability, close lattice matches to dopant ions and comparatively low phonon energy ${ }^{26}, \mathrm{GdVO}_{4}$ is a well-established host matrix for $\mathrm{Nd}^{3+}$, used in applications such as solid-state laser hosts and diode pumped microchip lasers ${ }^{27,28}$. In bioimaging applications, $\mathrm{GdVO}_{4}: \mathrm{Nd}$ has been investigated mainly in combination with other rare earth dopants for fluorescent up-conversion nanoparticles (UCNPs) ${ }^{26,29,30}$, a strategy often employed for avoiding the autofluorescence issue ${ }^{29}$. Conversely, this study is focused on single $\mathrm{Nd}$-doped $\mathrm{GdVO}_{4}$ for bioimaging applications thanks to the exploitation of in situ re-excitation and time-gated detection. For optimal clearance in in vivo applications, probe particles must be in the $30-150 \mathrm{~nm}$ size range ${ }^{31}$. Thus, the adopted synthetic procedure should guarantee a precise control over the particles' dimensions. Several synthetic strategies have been proposed for the preparation of $\mathrm{GdVO}_{4}$ nanoparticles doped with rare earth ions, including sol-gel ${ }^{32}$, urea hydrolysis ${ }^{33}$, electrospinning ${ }^{34}$, and hydro/solvothermal methods ${ }^{35,36}$. Inagaki et al. developed a synthesis to obtain $\mathrm{GdVO}_{4}: \mathrm{Nd} / \mathrm{Yb}$ nanoparticles with an homogenous dimensional distribution (around $20 \mathrm{~nm}$ ), but this approach 
requires a complex microreaction method followed by an autoclave treatment ${ }^{25}$. Kolesnikov et al. proposed a modified Pechini procedure for the synthesis of $\mathrm{YVO}_{4}: \mathrm{Nd} \mathrm{NPs}(40-50 \mathrm{~nm})$, but the nanoparticles were collected after a one day-long sedimentation, possibly leading to a low final yield $^{26}$. In this context, co-precipitation represents a cost-effective, experimentally simple and scalable alternative that can provide good control over particle size ${ }^{30}$. In the present comparative study, four synthetic procedures for the preparation of $\mathrm{GdVO}_{4}: \mathrm{Nd}$ based on the co-precipitation approach were selected and optimized: a simple co-precipitation was compared to colloidal, reverse micelles and sonochemical methods. The selected synthetic approaches started from the same lanthanide and vanadium sources and required no post-treatment at high temperature to promote crystallinity, but differed in the strategy adopted to ensure a morphological control over the prepared particles: from the use of chelating agents, to the use of micellar microreactors and ultrasounds. The role of the amount of added dopant was also investigated. The prepared materials were extensively characterized for their structural, morphological, surface and luminescence properties, also with respect to micrometric reference material, in order to clarify the role of the synthetic pathway on the imaging performance. It should be noted that, to the authors' best knowledge, this represents the first comparative study of different synthetic procedures for this material, as well as the first investigation of the role of $\mathrm{Nd}$ content in single doped $\mathrm{GdVO}_{4}$ nanoparticles.

\section{Experimental section}

\section{$2.1 \mathrm{GdVO}_{4}: \mathrm{Nd}$ syntheses}

All of the reactants were purchased from Sigma-Aldrich and employed without additional purification. Solutions and suspensions were prepared by using doubly distilled water passed through a Milli-Q apparatus.

Four different sample series were synthesized using different approaches: co-precipitation, colloidal, sonochemical and reverse micelles syntheses. In addition, a reference micrometric sample was prepared by a standard solid state synthesis. The $\mathrm{Nd}: \mathrm{Gd}$ molar ratio was varied from $0.5 \%$ to $10 \%$.

\subsubsection{Coprecipitation synthesis}

A co-precipitation method, previously reported for the formation of $\mathrm{GdVO}_{4}: \mathrm{Eu}^{30}$, was here optimised for the synthesis of $\mathrm{GdVO}_{4}: \mathrm{Nd}$. In a typical synthesis, $110 \mathrm{~mL}$ of a $0.05 \mathrm{M}$ aqueous solution of lanthanides $\left(\mathrm{Gd}\left(\mathrm{NO}_{3}\right)_{3} \cdot 6 \mathrm{H}_{2} \mathrm{O}\right.$ and $\left.\mathrm{Nd}\left(\mathrm{NO}_{3}\right)_{3} \cdot 6 \mathrm{H}_{2} \mathrm{O}\right)$ was added dropwise to $110 \mathrm{~mL}$ of a $0.05 \mathrm{M} \mathrm{NH}_{4} \mathrm{VO}_{3}$ aqueous solution, obtained by its dissolution in $\mathrm{NaOH} 0.15 \mathrm{M}$. The final solution was stirred at $60{ }^{\circ} \mathrm{C}$ for $60 \mathrm{~min}$. The precipitate was then separated by centrifugation and washed 
several times with MilliQ water until neutrality was reached; the collected product was dried at 60 ${ }^{\circ} \mathrm{C}$ for 20 hours. The as-prepared samples are denoted as $\mathrm{CP} x$, where $x$ is the nominal $\mathrm{Nd}: \mathrm{Gd}$ molar ratio percentage.

\subsubsection{Colloidal synthesis}

In a colloidal synthesis ${ }^{13}, 110 \mathrm{~mL}$ of a $0.05 \mathrm{M}$ sodium citrate aqueous solution was added dropwise to $147 \mathrm{~mL}$ of a $0.05 \mathrm{M}$ aqueous solution of lanthanide ions $\left(\mathrm{Gd}\left(\mathrm{NO}_{3}\right)_{3}\right.$ hexahydrate and $\mathrm{Nd}\left(\mathrm{NO}_{3}\right)_{3}$ hexahydrate). The white precipitated of lanthanide citrate complex was completely dissolved by dropwise addition of $110 \mathrm{~mL}$ of $0.05 \mathrm{M} \mathrm{NH}_{4} \mathrm{VO}_{3}$ aqueous solution, obtained by its dissolution in $\mathrm{NaOH} 0.15 \mathrm{M}$. The resulting solution was stirred at $60{ }^{\circ} \mathrm{C}$ for 60 minutes, during which the clear solution becomes bluish. Finally, the colloidal solution was cooled down to room temperature. Purification by dialysis (tubular dialysis membrane SpectraPor 4 Regenerate Cellulose mwco 12-14 $\mathrm{kD}$ ) against water enabled a slow growth of the particles until neutrality was reached (in about 6 days). Then, the solvent was removed in a ventilated oven at $70{ }^{\circ} \mathrm{C}$ for 20 hours. The samples obtained with this method are denoted as $\mathrm{C} x$ where $x$ is the nominal $\mathrm{Nd}$ : Gd molar ratio.

\subsubsection{Sonochemical synthesis}

The sonochemical method, proposed in the literature for the synthesis of pure $\mathrm{GdVO}_{4}{ }^{37}$, was here adapted for the formation of $\mathrm{GdVO}_{4}: \mathrm{Nd}$ : a lower sonication power and different stabilizing agent were here adopted and no final calcination was here performed. Initially, $2.433 \mathrm{~g}$ of $\mathrm{Gd}\left(\mathrm{NO}_{3}\right)_{3}$ hexahydrate, $0.048 \mathrm{~g}$ of $\mathrm{Nd}\left(\mathrm{NO}_{3}\right)_{3}$ hexahydrate and $0.643 \mathrm{~g}$ of $\mathrm{NH}_{4} \mathrm{VO}_{3}$ were dissolved in $55 \mathrm{~mL}$ of distilled water and placed in a $250 \mathrm{~mL}$ beaker. Subsequently, $6.1 \mathrm{~mL}$ of PEG-600 was added. The solution was then sonicated using a Thermo-Fischer Q700 ultrasonic processor working at $20 \mathrm{kHz}$, equipped with a $13 \mathrm{~mm}$ diameter Ti-6Al-4 V horn immersed for $c a .20 \mathrm{~mm}$. Sonication was conducted at $38 \mathrm{~W} \mathrm{~cm}^{-2}$ in an ice bath using a pulsed mode, with $10 \mathrm{~s}$ pulses separated by $10 \mathrm{~s}$ interval, for an overall duration of $30 \mathrm{~min}$ (including on and off time for pulsed sonication). Then, $0.43 \mathrm{~g}$ of $\mathrm{NaOH}$ was added and sonication was further continued for $150 \mathrm{~min}$, cooling the reaction mixture by a water/ice bath. The precipitate was collected by centrifugation and washed several times with a 50 : 50 water : ethanol mixture, in order to remove excess PEG-600 and base. The product was then dried overnight in a vacuum oven. The samples synthesised by the sonochemical procedure are named SCx, with $x$ being the nominal $\mathrm{Nd}: \mathrm{Gd}$ molar ratio.

\subsubsection{Reverse micelles synthesis}

In the literature, the reverse micelles method has been previously reported for the synthesis of $\mathrm{GdVO}_{4}: \mathrm{Eu}$ and $\mathrm{GdVO}_{4}: \mathrm{Er} / \mathrm{Yb}^{38}$. Herein, a reverse micelle solution was prepared by mixing an oil phase, formed by mixing cyclohexane $(100 \mathrm{~mL})$, Triton X-100 (60 mL) and $n$-pentanol $(20 \mathrm{~mL})$, with a $0.1 \mathrm{M} \mathrm{NH}_{4} \mathrm{VO}_{3}$ solution, prepared by dissolving the vanadate in a $10 \mathrm{~mL}$ of $0.15 \mathrm{M} \mathrm{NaOH}$ 
aqueous solution. Then, $10 \mathrm{~mL}$ of a $0.1 \mathrm{M}$ lanthanides aqueous solution $\left(\mathrm{Gd}\left(\mathrm{NO}_{3}\right)_{3}\right.$ hexahydrate and $\mathrm{Nd}\left(\mathrm{NO}_{3}\right)_{3}$ hexahydrate) was added dropwise and stirred continuously for $60 \mathrm{~min}$ at room temperature. Then, the solution was aged for 24 hours at room temperature, during which it turned yellowish. The solution was then destabilized by addition of methanol. The resulting precipitate was separated by centrifugation and washed with water : methanol $50: 50$ to remove the excess of surfactant. The powder was finally dried in a ventilated oven at $70{ }^{\circ} \mathrm{C}$ for 20 hours. Herein, the samples prepared by this method are labelled as $\mathrm{RM} x$, with $x$ being the nominal $\mathrm{Nd}: \mathrm{Gd}$ molar ratio.

\subsubsection{Solid state synthesis}

Reference micrometric samples were prepared by a solid state reaction ${ }^{39,40}$ using $\mathrm{NH}_{4} \mathrm{VO}_{3}, \mathrm{Gd}_{2} \mathrm{O}_{3}$ and $\mathrm{Nd}_{2} \mathrm{O}_{3}$ as starting materials. The precursors were mixed by grinding in an agate mortar. The obtained powder was then placed in a ceramic crucible and heated in a tubular furnace in air: a 300 ${ }^{\circ} \mathrm{C} / \mathrm{h}$ heating rate was adopted up to $1000{ }^{\circ} \mathrm{C}$, followed by a $2 \mathrm{~h}$ dwell time and a final $200{ }^{\circ} \mathrm{C} / \mathrm{h}$ cooling rate. After cooling, the powder was ground and washed with $2 \mathrm{M} \mathrm{NaOH}$ solution, distilled water and methanol. Solid state samples will be labelled as SSx where $x$ is the nominal $\mathrm{Nd}: \mathrm{Gd}$ molar ratio.

\subsection{Materials characterization}

Powder X-ray diffraction (PXRD) patterns were collected on a Bruker D5000-2 diffractometer working with $\mathrm{Cu} \mathrm{K} \alpha$ radiation $(0.154 \mathrm{~nm})$. Diffractograms were recorded with a step size of $0.02^{\circ}$ over the $2 \theta$ range $10-80^{\circ}$. Average crystallite sizes were estimated according to the Scherrer method:

$$
d=\frac{K \lambda}{\beta \cos \theta}
$$

on the line broadening $\beta$, corrected for instrumental broadening, of non-overlapping and nondegenerate reflections identified for all specimens, (200) and (101), using $\mathrm{K}=1$, as previously reported for similar systems ${ }^{19}$.

Elemental composition was investigated by energy-dispersive X-ray spectroscopy (EDX) on a Hitachi S-3400N scanning electron microscope.

Fourier transform infrared spectra (FT-IR) were collected on an ATR Perkin Elmer Spectrum 100 spectrometer according to a previously reported procedure ${ }^{41}$.

The particle morphology was investigated via transmission electron microscopy (TEM) using a 120 kV Zeiss LEO 912ab Energy Filtering microscope equipped with a CCD-BM/1 K system. High resolution TEM (HR-TEM) images were also acquired, using a field emission gun TEM microscope 
(FEI TECNAI F20), working at $200 \mathrm{kV}$ and equipped with S-Twin lens and of two CCD cameras (626 Gatan and 794 Gatan). Samples were deposited on 200 mesh Cu holey carbon grids after dispersion in isopropanol. Scanning electron microscopy (SEM) was also performed on selected samples, after sputtering with gold to counter charging effects, using an FEI Quanta 200 FEG SEM operating at high vacuum. For each electron microscopy image, the width and length of each individual nanoparticle were analysed adopting the Image J programme (see e.g. Figure S2 inset) and median values were determined.

Diffuse reflectance spectra (DRS) were recorded on a Perkin Elmer Lambda 1050 UV-Vis-NIR spectrophotometer in the 450-850 nm spectral range. Absorbance spectra were determined according to the Kubelka-Munk function, by assuming an isotropic distribution of reflections, a random distribution of particles, layer thickness much larger than particle size and a constant scattering coefficient in a certain energy range. ${ }^{42}$

Emission spectra were recorded on a fibre-coupled, cooled InGaAs array spectrometer (Avantes Avaspec-NIRS512-1.7-HSC-EVO). The excitation was carried out using a continuous $808 \mathrm{~nm} / 500$ $\mathrm{mW}$ diode laser (Roithner Lasertechnik). Excitation spectra were recorded using an Edinburgh Instruments FS920 photoluminescence spectrometer using a $450 \mathrm{~W}$ xenon lamp as the excitation source and a germanium detector.

Luminescence decay measurements were performed on a homemade setup consisting of a pulsed tuneable optical parametric oscillator laser (Ekspla NT342), tuned to $808 \mathrm{~nm}$ (10 Hz pulse rate, $5 \mathrm{~ns}$ pulse duration) and a Thorlabs PDA20C/M InGaAs amplified photodiode detecting wavelengths in the $800-1700 \mathrm{~nm}$ range, with a $5 \mathrm{MHz}$ bandwidth. The signal from the photodiode was displayed on a Rigol DS2302A $300 \mathrm{MHz}$ oscilloscope. Decay profiles were fitted manually including a background or zero offset term, $I_{B}$, in the fitting function to correct for a non-zero signal when no light was measured:

$$
I(t)=I_{01} \cdot e^{-t / \tau_{1}}+I_{02} e^{-t / \tau_{2}}+I_{B}
$$

The average decay time was calculated as follows:

$$
\tau_{\text {avg }}=\frac{I_{01} \tau_{1}+I_{02} \tau_{2}}{I_{01}+I_{02}}
$$

The fraction of the short component was calculated according to the equation:

$$
F_{\text {short }}=\frac{I_{02} \tau_{2}}{I_{01} \tau_{1}+I_{02} \tau_{2}}
$$

\section{Results and discussion}


The structural properties of $\mathrm{GdVO}_{4}: \mathrm{Nd}$ samples were determined via PXRD. Diffractograms of 2\% Nd-doped samples are compared in Figure 1. All the analysed samples reveal a single zircon-type phase of $\mathrm{GdVO}_{4}$ : a body-centred tetragonal crystal structure, corresponding to space group I4//amd. No other peaks are observed, indicating the absence of crystalline impurities. In particular, no peaks related to segregated phases of neodymium oxides are appreciable, also at the highest tested $\mathrm{Nd}$ content (Figure S1), supporting a successful integration of $\mathrm{Nd}^{3+}$ ions into the $\mathrm{GdVO}_{4}$ host lattice. In good agreement with previous reports about micrometric $\mathrm{GdVO}_{4}: \mathrm{Nd}^{43}$, any shift in the peak position as a function of $\mathrm{Nd}$ doping is hardly appreciable, due to the similar ionic radii and ionic charges of $\mathrm{Nd}^{3+}$ and $\mathrm{Gd}^{3+}$ ions (0.112 and $0.105 \mathrm{~nm}$, respectively).

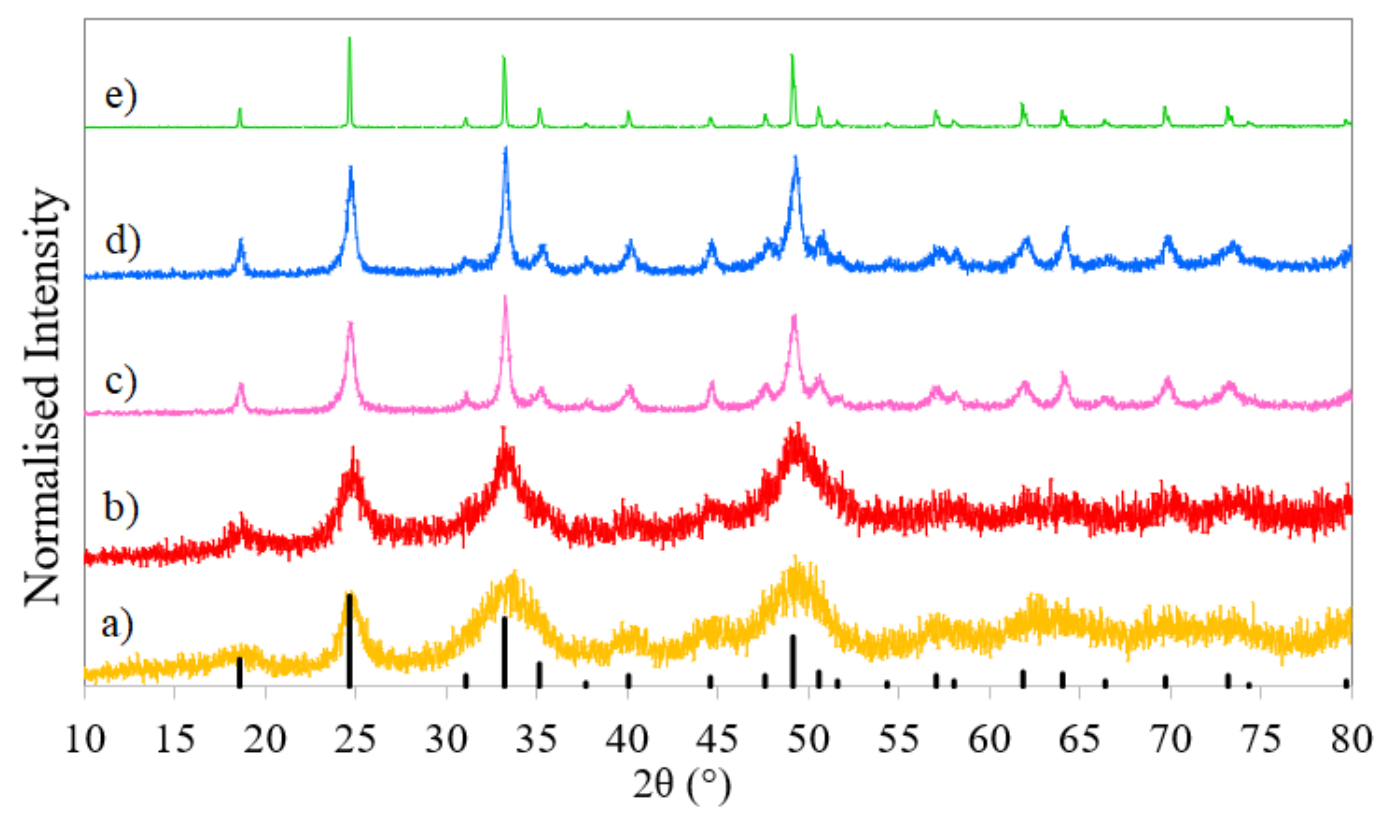

Figure 1 - PXRD patterns of (a) C2, (b) RM2, (c) CP2, (d) SC2 and (e) SS2 phosphors. GdVO 4 diffraction pattern from ICSD (No. 15607) is also reported as reference.

Figure 1 clearly shows a different degree of peak broadening for each sample, indicating a dependence of crystallite dimensions on the synthetic procedure. In particular, while the solid state synthesis shows very sharp and well defined peaks, the colloidal and reverse micelles procedures lead to patterns characterized by broad and overlapping peaks; the co-precipitation and sonochemical approaches show an intermediate behaviour, presenting clearly defined peaks but with appreciable peak broadening. Table 1 lists the crystallite sizes determined by Scherrer equation on the (200) and (101) reflections. Notwithstanding the many limitations of the Scherrer approach, it can be used to provide an estimate of the crystallite dimensions in the direction perpendicular to the planes that produced the diffraction peak. In order to compare the crystallite dimensions of the different samples, the peak broadening of two non-overlapping and non-degenerate reflections 
identified for all specimens was considered; the resulting values were also representative of the maximum and minimum crystallite sizes estimated by analysing all of the reflections present in the individual diffractograms. A direction dependence of the crystallite dimensions can be inferred. The colloidal and the reverse micelles methods gave rise to the smallest crystallites (close to $10 \mathrm{~nm}$ ), which can be traced back to the chelating effect of sodium citrate and to the enclosed reaction environment, respectively. On the contrary, the sonochemical procedure resulted in the largest crystallites among the wet syntheses: ultrasound-assisted synthesis can promote crystal growth due to the microthermal heating generated out of sonication energy ${ }^{44}$.

Table 1 - Crystallite dimensions evaluated with Scherrer method on different diffractogram peaks of $2 \% \mathrm{Nd}$ doped samples, together with median particle length, width and aspect ratio estimated by electron microscopy images.

\begin{tabular}{|c|c|c|c|c|c|}
\hline \multirow{2}{*}{ Sample } & \multicolumn{2}{|c|}{ Crystallite dimension (nm) } & \multicolumn{2}{|c|}{ Particle size (nm) } & \multirow{2}{*}{ Aspect ratio } \\
\hline & $d(200)$ & $d(101)$ & width & length & \\
\hline $\mathrm{CP} 2$ & 25 & 56 & 41 & 110 & 2.7 \\
\hline $\mathrm{C} 2$ & 10 & 15 & 7 & 14 & 2.0 \\
\hline $\mathrm{SC} 2$ & 27 & 86 & 33 & 64 & 2.0 \\
\hline RM2 & - & 13 & 6 & 8 & 1.3 \\
\hline SS2 & $>100$ & $>100$ & 1522 & 1266 & 1.2 \\
\hline
\end{tabular}

The morphology of the prepared particles was investigated by TEM investigations. Figure 2 reports TEM images of $2 \% \mathrm{Nd}$-doped samples. All samples display nanometric particles with an elongated, spindle-like morphology, which is irrespective of the type of adopted synthesis. Similar elongated morphologies have been previously reported for $\mathrm{GdVO}_{4}$ materials ${ }^{23,30,37}$. The only notable exception is the sample from solid state synthesis, which shows micrometric, polyhedral particles (Figure S2). In this respect, Jovanovic et al. have reported a change in morphology of GdVO 4 :Eu from nanorods to faceted single crystals of round and rectangular shape upon annealing at $1000{ }^{\circ} \mathrm{C}^{30}$.

Table 1 reports the estimated particle lengths and aspect ratio, estimated on a set of independent TEM images for each sample. Data in Table 1 show quite good agreement between particle dimensions evaluated by TEM images and average crystallite size values obtained by means of Scherrer equation. Notwithstanding the limitations of the Scherrer approach, the fact that estimated crystallite dimensions and particle sizes are in good agreement supports the absence of internal substructures. This conclusion is also confirmed by HR-TEM images, showing elongated nanoparticles with well-distinguishable fringe patterns (Figure 2b, inset). The highly ordered habit of nanoparticles confirms the high crystallinity of the materials, despite the absence of a high 
temperature step during the synthesis. It should be noted that well crystalline particles are observed also for the colloidal synthesis. This result could be attributed to the dialysis synthetic step, which allowed a controlled growth of the crystallites through the slow change in the solution $\mathrm{pH}$. As a matter of fact, the $\mathrm{pH}$ of the medium has a strong impact on the formation of the phosphor particles $^{30}$.

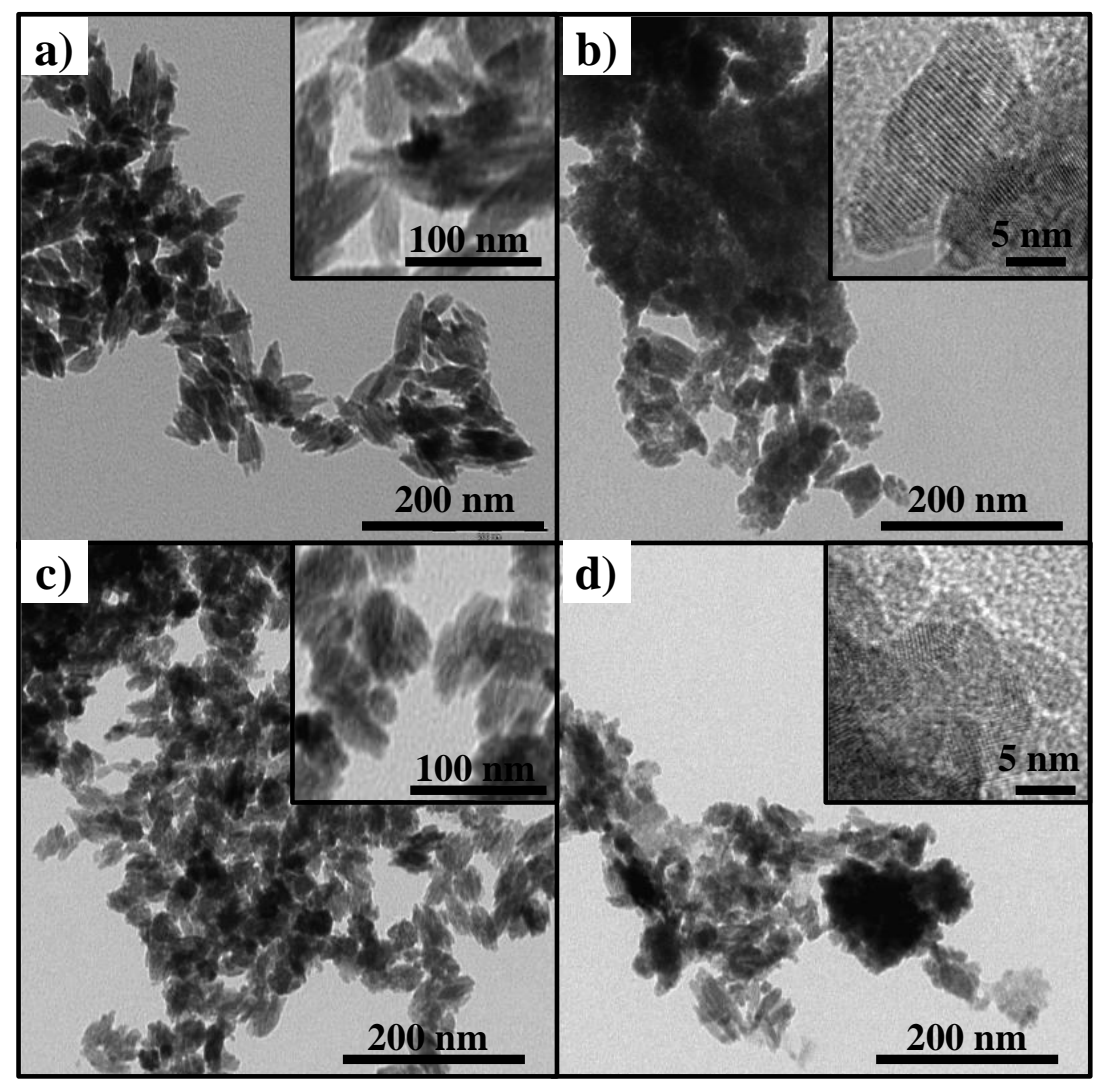

Figure 2 - (HR)TEM images of (a) CP2, (b) C2, (c) SC2 and (d) RM2 nanoparticles.

EDX mapping experiments highlighted the homogenous dispersion of $\mathrm{Nd}^{3+}$ ions in the samples. Experimental results are quite comparable with the nominal value of $\mathrm{Nd}$ as well as with the nominal $\mathrm{Gd} / \mathrm{V}$ molar ratio (Table S1). In particular, at the lower tested concentrations, the $\mathrm{Nd}$ content is consistent with the nominal amount, within the limits of the characterization technique (Table S1). However, at the highest $\mathrm{Nd}$ precursor concentration, the detected $\mathrm{Nd}$ content is significantly lower than the nominal amount: when a higher amount of $\mathrm{Nd}$ is added, not all of it is properly incorporated in the host lattice, as previously reported ${ }^{40}$. It is noteworthy that CP2 and SC2 samples show the Gd/V molar ratio closest to unity (respectively 1.09 and 1.10), while C2 and RM2 are characterized by a slightly higher Gd content (1.40 and 1.41). This result mirrors FTIR results (vide infra). In addition, EDX spectra showed the presence of impurity elements in three out of five samples (Table S2). In colloidal samples, sodium impurities remain from the reactants (sodium citrate and sodium hydroxide), which were not completely removed by dialysis. A slight titanium 
contamination in the sonochemical sample is ascribable to microtip damage used during the prolonged ultrasound irradiation.

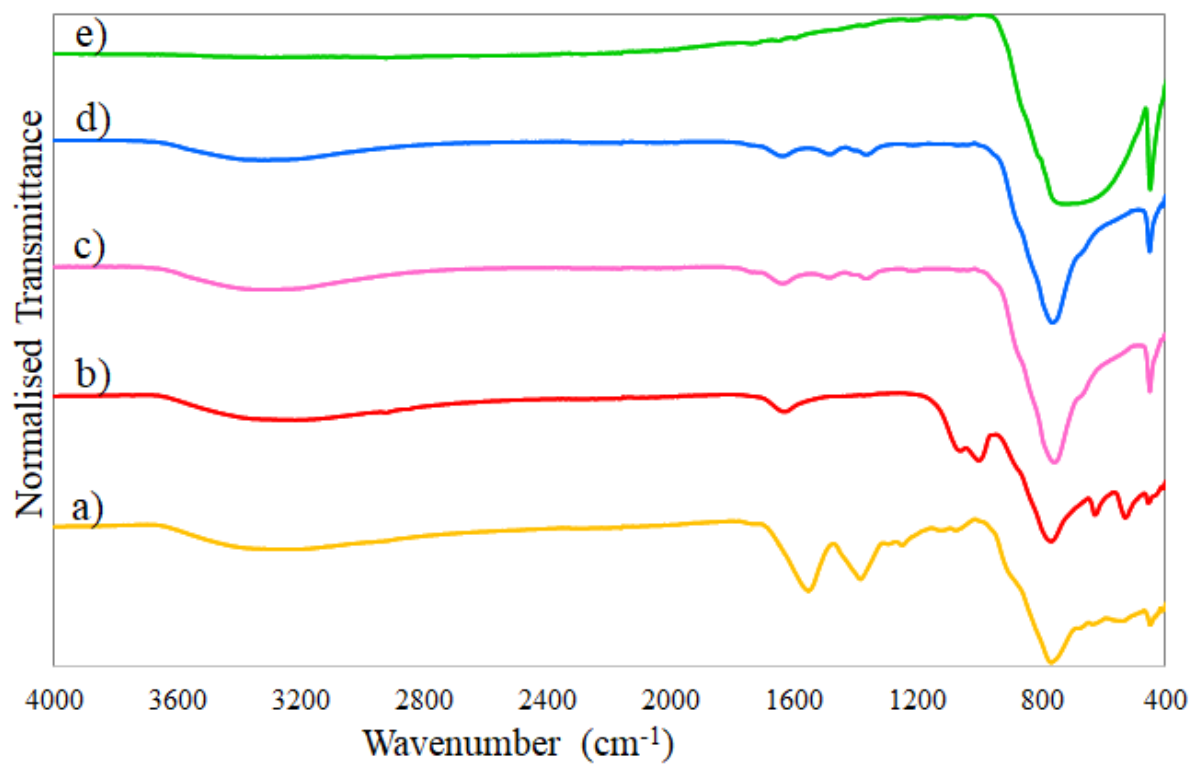

Figure 3 - FT-IR spectra of (a) C2, (b) RM2, (c) CP2, (d) SC2 and (e) SS2.

Luminescence properties are strongly affected by the presence of impurities. In this respect, FTIR spectroscopy represents a valuable tool ${ }^{7}$, which enables to detect also amorphous contaminants not appreciable by PXRD. FTIR spectra of $2 \%$ doped samples are reported in Figure 3. The formation of $\mathrm{GdVO}_{4}$ is confirmed by the two characteristic bands at around 765 and $451 \mathrm{~cm}^{-1}$ that can be attributed to the Gd-O and V-O stretching vibrations of the host lattice, respectively ${ }^{30,45}$. All of the nanometric samples show bands indicative of surface hydroxylation: the broad envelope centred at around $3300 \mathrm{~cm}^{-1}$ can be ascribed to the stretching vibration of hydroxyl groups mutually interacting by hydrogen bonding; its spectroscopic partner, located at $1630 \mathrm{~cm}^{-1}$, is attributed to in plane $\mathrm{HOH}$ bending mode of undissociated water molecules ${ }^{46}$. On the contrary, the spectrum of SS2 does not show these components, owing to its high temperature synthesis. It is worth noting that a poorly hydrophilic surface is indicative of low colloidal stability in aqueous media ${ }^{47}$.

In addition, the spectrum of the $\mathrm{C} 2$ sample shows two intense bands at 1383 and $1570 \mathrm{~cm}^{-1}$. These signals correspond to the symmetrical and asymmetrical vibrations of carboxyl groups ascribable to the presence of sodium citrate residues ${ }^{48}$. This finding is in agreement with EDX measurements showing $\mathrm{Na}$ impurities for colloidal samples ${ }^{49}$. Furthermore, the RM2 spectrum is characterised by bands at around 557, 628, 1000 and $1023 \mathrm{~cm}^{-1}$ which correspond to $\mathrm{V}-\mathrm{O}$ and $\mathrm{V}-\mathrm{O}-\mathrm{V}$ vibration modes ${ }^{50}$, supporting the presence of amorphous $\mathrm{V}_{2} \mathrm{O}_{5}$ phase in the sample.

Samples were also characterized for their optical and luminescence properties. In all the absorption spectra (Figure S3), two main peaks are clearly visible: the first one at $c a .595 \mathrm{~nm}$ and the second 
one at $808 \mathrm{~nm}$. Moreover, some samples show a wide absorption band at wavelengths lower than $500 \mathrm{~nm}$. This broad band can be attributed to the presence of amorphous $\mathrm{V}_{2} \mathrm{O}_{5}$ on the grounds of the absorption spectrum of $\mathrm{V}_{2} \mathrm{O}_{5}$ (Figure $\mathrm{S} 3 \mathrm{~b}$ ). Based on the intensity of this absorption band, the $\mathrm{V}_{2} \mathrm{O}_{5}$ amount can be estimated to increase in the order: $\mathrm{SC} 2<\mathrm{C} 2<\mathrm{RM} 2$. It is noteworthy that $\mathrm{CP} 2$ composite does not present any absorption below $550 \mathrm{~nm}$. Conversely, RM2 sample is characterized by an absorption spectrum completely dominated by the presence of $\mathrm{V}_{2} \mathrm{O}_{5}$ broad band, confirming the FT-IR and EDX findings.

Photoluminescence spectroscopy measurements were carried out to evaluate and compare the excitation spectra as well as the emission profiles and intensities of the prepared samples. As a representative example, Figure 4 reports the excitation and emission spectra of sample CP1; the electronic transitions related to each peak are highlighted. For excitation spectra, the emission wavelength was set at $1063 \mathrm{~nm}$. Two main excitation peaks were observed at 595 and $808 \mathrm{~nm}$, as in the absorption spectra, and are related to ${ }^{4} \mathrm{I}_{9 / 2} \rightarrow{ }^{4} \mathrm{G}_{5 / 2}$ and to ${ }^{4} \mathrm{I}_{9 / 2} \rightarrow{ }^{4} \mathrm{~F}_{5 / 2}+{ }^{2} \mathrm{H}_{9 / 2}$ electronic transitions, respectively. ${ }^{51}$ Albeit the two peaks appear to be equally intense, the $808 \mathrm{~nm}$ component $\left({ }^{4} \mathrm{I}_{9 / 2} \rightarrow{ }^{4} \mathrm{~F}_{5 / 2}+{ }^{2} \mathrm{H}_{9 / 2}\right.$ transition $)$ is the one of major interest because it lies in the BW-I and corresponds to the emission of diode lasers ${ }^{18}$. Emission spectra were recorded using an excitation wavelength of $808 \mathrm{~nm}$. All the emission spectra (Figure S4) exhibit the three emission peaks characteristic of Nd-doped materials. Among them, the most intense feature lies at around $1062 \mathrm{~nm}$ $\left({ }^{4} \mathrm{~F}_{3 / 2} \rightarrow{ }^{4} \mathrm{I}_{11 / 2} \text { transition }\right)^{14}:$ this is an ideal emission wavelength because it lies in the BW-II's range where tissue autofluorescence presents a minimum.
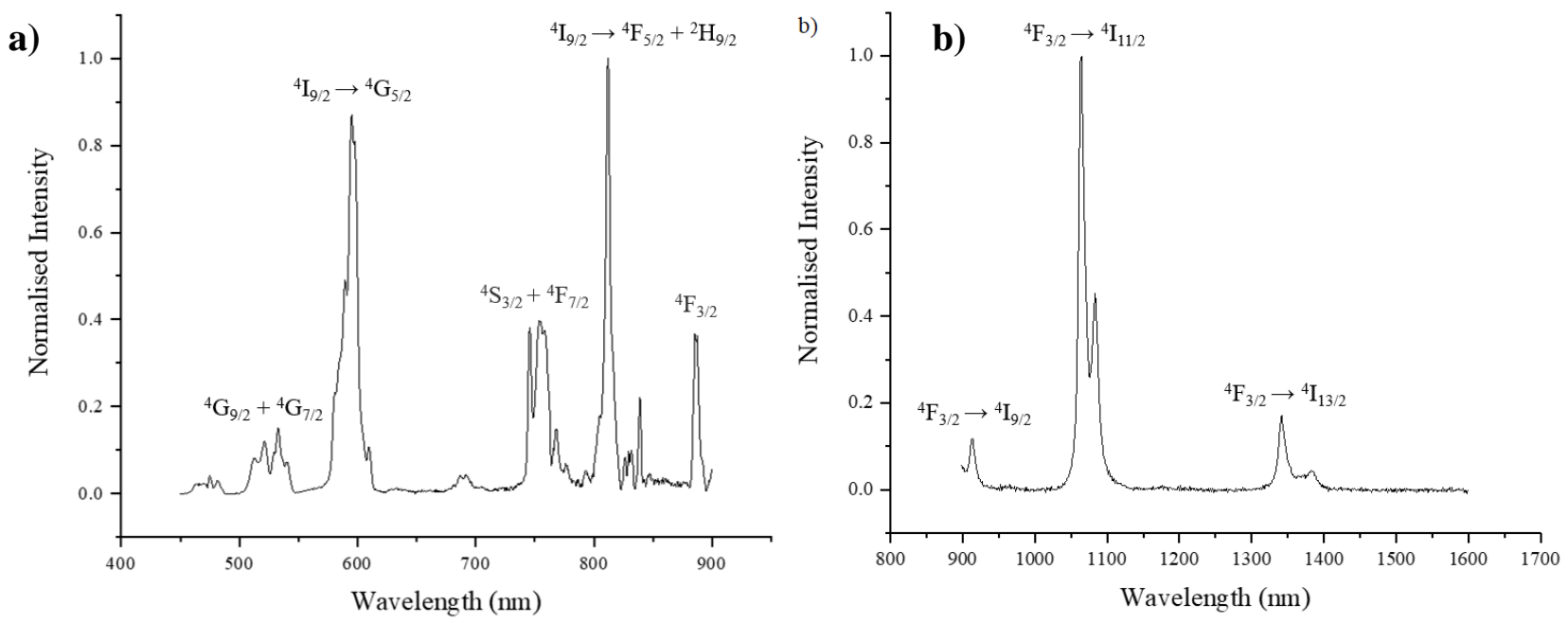

Figure 4 - Excitation (a) and emission (b) spectra of $G d V O_{4}: N d$ with relative electronic transitions of CP1. 
Figure 5a compares the normalised emission intensities of the synthesized NPs. Samples C2 and CP2 are characterized by the most intense emission, while a less than halved intensity is shown by RM2 and SC2. This trend cannot be correlated with the purity of the samples, neither with their particle dimensions. In this respect, the solid state reference shows an emission intensity one order of magnitude higher than the tested NPs (data not shown): high temperature annealing has been previously reported to promote the emission intensity of rare earth doped $\mathrm{GdVO}_{4}{ }^{30}$ due to higher crystallinity. It should be noted that sample C2, despite its small crystallite size, displays highly ordered crystalline habit, as shown in HR-TEM images where individual particles with well defined fringes are appreciable. The high crystallinity of this sample could be related to the slow growth adopted during synthesis, which was made possible by the use of citrate stabilizer and of the dialysis step. It should be noted that, the RM2 sample, which displays similar crystallite and particle size to $\mathrm{C} 2$, presents a lower crystallinity degree as apparent by HR-TEM images. This evidence is reflected in the emission intensity results, where RM2 shows a much lower performance than $\mathrm{C} 2$.

The role of the Nd content was investigated on the two best performing syntheses: the colloidal and co-precipitation routes. Figure S5 reports the emission spectra of the CP series as a representative example. The emission intensity increases up to $2 \%$, then at $10 \%$ a decrease is observed due to concentration quenching. This finding is in agreement with previous reports about micrometric $\mathrm{GdVO}_{4}$, showing an optimal Nd content around $2 \%{ }^{39,40}$.
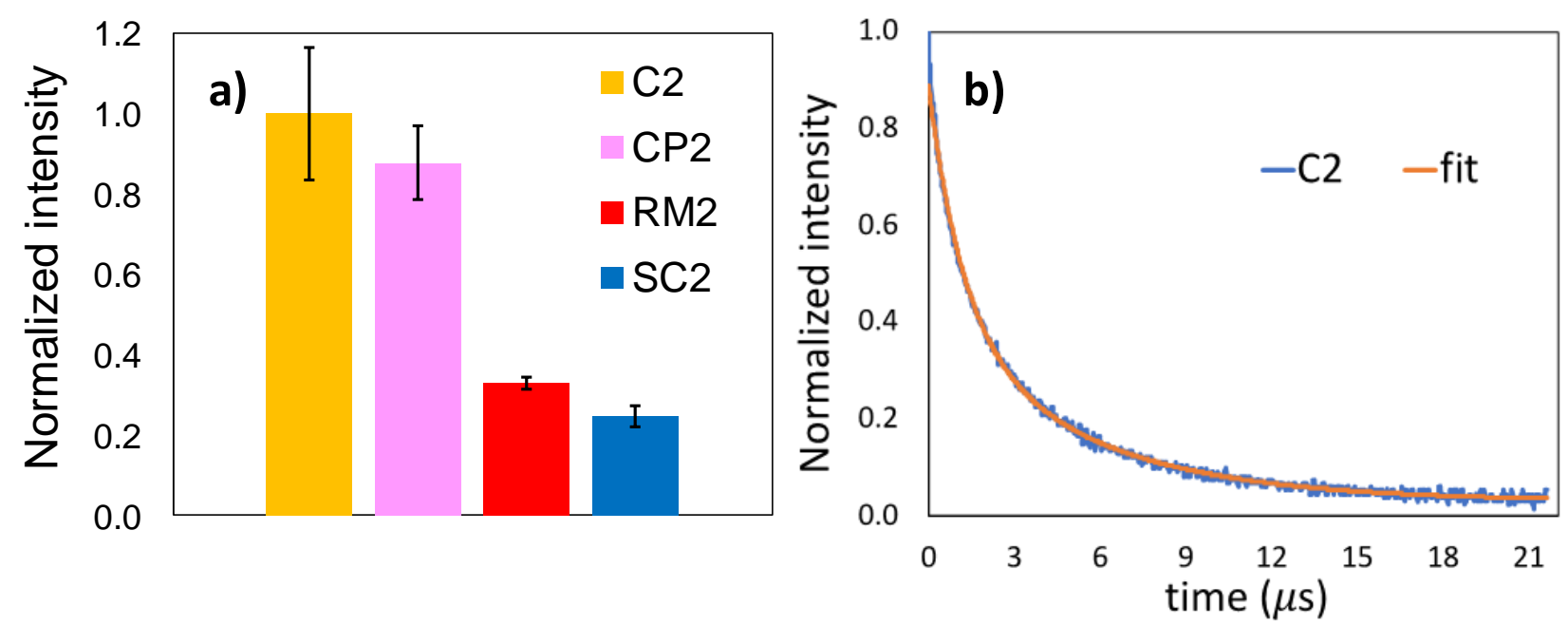

Figure 5 -a) Integrated peak counts emission intensities of $2 \%$ Nd-doped NPs; data are normalised on the highest value of the C2 sample intensity. b) Decay measurement of the $1060 \mathrm{~nm}$ emission peak of C 2 sample and its fit.

Finally, the luminescence decay of the synthesized samples was investigated. For the application of $\mathrm{GdVO}_{4}: \mathrm{Nd}$ in time-gated imaging, the decay time must be longer than autofluorescence decay 
lifetime, which is in the order of the nanoseconds ${ }^{52}$. Decay measurements were performed over the 800-1700 nm range of wavelengths and decay profiles were fitted using a double exponential function. It is quite common in luminescent material to observe more than one exponential decay component. While hard to prove, this is usually attributed to part of the dopants in a different (nonideal) environment, close to a defect or another dopant. A representative example is reported in Figure 5b, while the decay curves of the other samples are reported in Figure S6. The determined decay times for all the $2 \%$ doped samples are reported in Table 2. The solid state sample shows the longest decay lifetime, with values quite comparable to literature reports about micrometric crystals with similar dopant content ${ }^{27,53}$. The nanosized samples present faster decay, with $\mathrm{C} 2$ showing the longer decay lifetime among them. The longer decay time of $\mathrm{C} 2$, pointing to a lower degree of defect-related non-radiative decay, is in correspondence with its higher emission intensity noted before. However, it should be noted that all of the synthesized samples exhibit lifetimes significantly longer than autofluorescence.

The effect of dopant concentration on decay curves was also investigated (Figure S7). Lifetime values decrease with increasing $\mathrm{Nd}^{3+}$ content (Table S3), supporting the occurrence of concentration quenching where the excited energy has a higher probability to decay nonradiatively. ${ }^{30}$

Table 2 - Decay times of $2 \%$ Nd-doped samples.

\begin{tabular}{ccccc}
\hline sample & $\begin{array}{c}\boldsymbol{\tau}_{\boldsymbol{I}} \\
(\boldsymbol{\mu})\end{array}$ & $\begin{array}{c}\boldsymbol{\tau}_{\mathbf{2}} \\
(\boldsymbol{\mu} \boldsymbol{s})\end{array}$ & $\begin{array}{c}\text { fraction short } \\
\text { component }(\%)\end{array}$ & $\begin{array}{c}\text { Average decay } \\
\text { time }(\boldsymbol{\mu s})\end{array}$ \\
\hline $\mathrm{CP} 2$ & 2.8 & 0.6 & 13 & 1.9 \\
\hline $\mathrm{C} 2$ & 5.2 & 1.2 & 23 & 2.9 \\
\hline $\mathrm{SC} 2$ & 3.8 & 0.7 & 14 & 2.3 \\
\hline $\mathrm{RM} 2$ & 2.9 & 0.5 & 17 & 1.6 \\
\hline $\mathrm{SS} 2$ & 57 & 4.8 & 3.8 & 40 \\
\hline
\end{tabular}

In Table 3, the physicochemical properties of the colloidal $\mathrm{GdVO}_{4}: \mathrm{Nd}$ samples are compared to the ones of other nanophosphors proposed for time-gated in vivo bioimaging by recent literature studies. It is noteworthy that several of the reported nanosystems present excitation wavelengths in the UV and visible region not suitable for in vivo excitation. Our samples favourably compare with other phosphors also in terms of emission wavelength since it falls within the 1050-1150 nm window, where autofluorescence drops, making it a better suited material for deep tissue imaging. Furthermore, our materials are simpler and easier to synthesize than most of the complex systems 
reported in the literature and their luminescence lifetimes are much longer than tissue autofluorescence.

Table 3 - Comparison with other nanomaterials for time-gated in vivo bioimaging.

\begin{tabular}{|c|c|c|c|c|c|}
\hline nanosystem & $\begin{array}{l}\text { average size } \\
(\mathbf{n m})\end{array}$ & $\begin{array}{c}\text { excitation } \\
\text { wavelength } \\
(\mathbf{n m})\end{array}$ & $\begin{array}{c}\text { emission } \\
\text { wavelength } \\
(\mathbf{n m})\end{array}$ & $\begin{array}{l}\text { lifetime } \\
\qquad(\mu s)\end{array}$ & reference \\
\hline Si nanocrystals & 5 & 365 & 945 & 120 & {$[54]$} \\
\hline Si nanoparticles & 159 & 370 & 650 & 12 & {$[52]$} \\
\hline $\mathrm{CuInSe}_{2} / \mathrm{ZnS}$ & 32 & 425 & 695 & - & {$[55]$} \\
\hline ZnCuInSe/ZnS & 4 & 659 & 800 & $0.15-0.3$ & {$[56]$} \\
\hline $\mathrm{NaGdF}_{4}: \mathrm{Nd}^{3+}$ & 520 & 808 & 1060 & $\approx 100$ & {$[3]$} \\
\hline $\mathrm{NaYF}_{4}: \mathrm{Yb}, \mathbf{N d} @ \mathrm{CaF}_{2}$ & $6-8$ & 808 & 980 & $100-1500$ & {$[57]$} \\
\hline $\begin{array}{c}\mathrm{NaGdF}_{4}: 2 \% \mathrm{Yb}^{3+} \\
3 \% \mathrm{Nd}^{3+}, 0.2 \% \mathrm{Tm}^{3+}\end{array}$ & 13 & 790 & $980-1060$ & 1350 & [9] \\
\hline $\begin{array}{c}\mathrm{NaYF}_{4}: 10 \% \mathrm{Yb}^{3+} \\
30 \% \mathrm{Nd}^{3+} @ \mathrm{CaF}_{2}\end{array}$ & 9 & 800 & $980-1011$ & 833 & [9] \\
\hline $\mathrm{GdVO}_{4}: \mathrm{Nd}^{3+}, \mathrm{Yb}^{3+}$ & 20 & 600 & 985 & $63-143$ & {$[25]$} \\
\hline $\mathrm{GdVO}_{4}: \mathrm{Nd}^{3+}$ colloidal & $7-14$ & 808 & 1060 & $1.1-6.6$ & present study \\
\hline
\end{tabular}

\section{Conclusions}

In this work, four different synthetic procedures (colloidal, coprecipitation, sonochemical and reverse micelle methods) were compared for the preparation of $\mathrm{GdVO}_{4}: \mathrm{Nd} \mathrm{NPs}$, as NIR-emitting candidates for time-gated bioimaging. All synthetic procedures gave rise to crystalline $\mathrm{GdVO}_{4}$, even without high temperature treatments. A successful integration of $\mathrm{Nd}^{3+}$ was observed up to $2 \%$ nominal content, above which lower actual dopant amounts were observed. Elemental analysis, FTIR and absorption spectroscopies showed the occurrence of several amorphous impurities, with the only exception of samples from the co-precipitation route. All the synthetic procedures led to nanosized samples (smaller than $150 \mathrm{~nm}$ ), with spindle-like morphology and crystallite sizes comparable to the particle size observed via TEM. The adopted synthetic route enables a fine control over the particle size: from $60-100 \mathrm{~nm}$ for the sonochemical and co-precipitations methods, 
to close to $10 \mathrm{~nm}$ in the case of the reverse micelle and colloidal approaches. The reference solid state synthesis leads instead to polyhedral particles with lower aspect ratio and micrometric size. All the prepared phosphors presented the characteristic two major absorption peaks at 595 and $808 \mathrm{~nm}$ and a main emission at $1062 \mathrm{~nm}$. While the solid state reference showed very intense emission and longer decay times, the nanosized samples showed fluorescence lifetimes exceeding autofluorescence decay times and the co-precipitation and colloidal routes gave rise to nanosized samples with the most intense emission, possibly as a result of a higher degree of crystallinity. The effect of Nd content was also investigated on the best performing series, showing an optimal actual dopant content close to $2 \%$ to maximize emission intensity and maintain sufficiently long decay lifetimes.

\section{Acknowledgements}

Prof. Fernando Camara, Dr. Nicola Rotiroti, Nadia Santo and UNITECH COSPECT (Università degli Studi di Milano, Piattaforme Tecnologiche di Ateneo, Via Golgi 19, 20133 Milano) are kindly acknowledged for TEM analyses. T.C. acknowledges financial support from Erasmus+ program.

\section{References}

1. del Rosal, B., Villa, I., Jaque, D. \& Sanz-Rodríguez, F. In vivo autofluorescence in the biological windows: the role of pigmentation. J. Biophotonics 9, 1059-1067 (2016).

2. Jaque, D. et al. Inorganic nanoparticles for optical bioimaging. Adv. Opt. Photonics 8, 1 (2016).

3. del Rosal, B. et al. Overcoming Autofluorescence: Long-Lifetime Infrared Nanoparticles for Time-Gated In Vivo Imaging. Adv. Mater. 28, 10188-10193 (2016).

4. Hemmer, E., Benayas, A., Légaré, F. \& Vetrone, F. Exploiting the biological windows: current perspectives on fluorescent bioprobes emitting above $1000 \mathrm{~nm}$. Nanoscale Horizons 1, 168-184 (2016).

5. Berezin, M. Y. \& Achilefu, S. Fluorescence Lifetime Measurements and Biological Imaging. Chem. Rev. 110, 2641-2684 (2010).

6. le Masne de Chermont, Q. et al. Nanoprobes with near-infrared persistent luminescence for in vivo imaging. Proc. Natl. Acad. Sci. 104, 9266-9271 (2007).

7. Meroni, D., Porati, L., Demartin, F. \& Poelman, D. Sol-Gel Synthesis of CaTiO 3 :Pr $3+$ Red Phosphors: Tailoring the Synthetic Parameters for Luminescent and Afterglow Applications. ACS Omega 2, 4972-4981 (2017).

8. Cubeddu, R., Canti, G., Taroni, P. \& Valentini, G. Time-Gated Fuorescence Imaging For The 
Diagnosis Of Tumors In A Murine Model. Photochem. Photobiol. 57, 480-485 (1993).

9. Tan, M. et al. Rare-earth-doped fluoride nanoparticles with engineered long luminescence lifetime for time-gated in vivo optical imaging in the second biological window. Nanoscale 10, 17771-17780 (2018).

10. Poelman, D., Heggen, D. Van der, Du, J., Cosaert, E. \& Smet, P. F. Persistent phosphors for the future: fit for the right application. J. Appl. Phys.

11. Rajendran, M. \& Miller, L. W. Evaluating the Performance of Time-Gated Live-Cell Microscopy with Lanthanide Probes. Biophys. J. 109, 240-248 (2015).

12. Sukul, P. P. \& Kumar, K. Near-infrared (808 and $980 \mathrm{~nm}$ ) excited photoluminescence study in Nd-doped Y 2 O 3 phosphor for bio-imaging. Methods Appl. Fluoresc. 4, 044005 (2016).

13. del Rosal, B. et al. Neodymium-doped nanoparticles for infrared fluorescence bioimaging: The role of the host. J. Appl. Phys. 118, 143104 (2015).

14. Anderson, F. G., Summers, P. L., Weidner, H., Hong, P. \& Peale, R. E. Interpretive crystalfield parameters: Application to Nd3+ in GdVO4 and YVO4. Phys. Rev. B 50, 14802-14808 (1994).

15. Herault, E., Balembois, F. \& Georges, P. Nd:GdVO4 as a three-level laser at $879 \mathrm{~nm}$. Opt. Lett. 31, 2731 (2006).

16. Sun, X. et al. Passively Q-Switched Nd:GdVO4 1.3 $\mu \mathrm{m}$ Laser with Few-Layered Black Phosphorus Saturable Absorber. IEEE J. Sel. Top. Quantum Electron. 24, 1-5 (2018).

17. Singh, A. J., Sharma, S. K., Mukhopadhyay, P. K. \& Oak, S. M. Dual wavelength operation in diode-end-pumped hybrid vanadate laser. Pramana 75, 929-934 (2010).

18. Liang, H. et al. Wavelength dependence of cell cloning efficiency after optical trapping. Biophys. J. 70, 1529-1533 (1996).

19. Lee, N. et al. Iron Oxide Based Nanoparticles for Multimodal Imaging and Magnetoresponsive Therapy. Chem. Rev. 115, 10637-10689 (2015).

20. ICNIRP. International Commission on Non-Ionizing Radiation Protection ICNIRP Guidelines on Limits of Exposure to Incoherent Visible and Infrared Radiation. Health Phys. 105, 74-96 (2013).

21. Barolet, D., Christiaens, F. \& Hamblin, M. R. Infrared and skin: Friend or foe. J. Photochem. Photobiol. B Biol. 155, 78-85 (2016).

22. IEC International Electrotechnical Commision. Norme Internationale International Standard. European Standard, CENELEC, European Committee for Electrotechnical Standardization 2000, (2006).

23. Abdesselem, M. et al. Multifunctional Rare-Earth Vanadate Nanoparticles: Luminescent 
Labels, Oxidant Sensors, and MRI Contrast Agents. ACS Nano 8, 11126-11137 (2014).

24. Aime, S., Botta, M. \& Terreno, E. Gd(III)-Based Contrast Agents For MRI. in Advances in Inorganic Chemistry 173-237 (2005). doi:10.1016/S0898-8838(05)57004-1

25. Inagaki, T., Ishigaki, T. \& Ohmi, K. Near-infrared-emitting GdVO 4 :Nd,Yb nanophosphors synthesized by microreaction method for biological imaging. Jpn. J. Appl. Phys. 57, 112601 (2018).

26. Kolesnikov, I. E., Golyeva, E. V., Kurochkin, M. A., Lähderanta, E. \& Mikhailov, M. D. Nd3+-doped YVO4 nanoparticles for luminescence nanothermometry in the first and second biological windows. Sensors Actuators B Chem. 235, 287-293 (2016).

27. Jensen, T. et al. Spectroscopic characterization and laser performance of diode-laser-pumped Nd: GdVO4. Appl. Phys. B Lasers Opt. 58, 373-379 (1994).

28. Wyss, C. P. et al. Performance of a diode-pumped $5 \mathrm{~W} \mathrm{Nd} \mathrm{3+:GdVO} 4$ microchip laser at $1.06 \mu \mathrm{m}$. Appl. Phys. B Lasers Opt. 68, 659-661 (1999).

29. Chen, X., Liu, Y. \& Tu, D. Lanthanide-Doped Luminescent Nanomaterials. (Springer Berlin Heidelberg, 2014). doi:10.1007/978-3-642-40364-4

30. Jovanović, D. J. et al. Annealing effects on the microstructure and photoluminescence of Eu 3+-doped GdVO4 powders. Opt. Mater. (Amst). 35, 1797-1804 (2013).

31. Gaumet, M., Vargas, A., Gurny, R. \& Delie, F. Nanoparticles for drug delivery: The need for precision in reporting particle size parameters. Eur. J. Pharm. Biopharm. 69, 1-9 (2008).

32. Liu, G., Hong, G., Wang, J. \& Dong, X. Deposition of GdVO 4 :Eu 3+ nanoparticles on silica nanospheres by a simple sol-gel method. Nanotechnology 17, 3134-3138 (2006).

33. Singh, N. S. et al. Luminescence study of Eu3+ doped GdVO4 nanoparticles: Concentration, particle size, and core/shell effects. J. Appl. Phys. 104, 104307 (2008).

34. Li, X. et al. One-dimensional GdVO4:Ln3+ (Ln=Eu, Dy, Sm) nanofibers: Electrospinning preparation and luminescence properties. J. Solid State Chem. 184, 141-148 (2011).

35. Kim, M.-J. \& Huh, Y.-D. Preparation and Photoluminescence of GdVO 4 :Eu Nanophosphors for Flexible and Transparent Displays. Bull. Korean Chem. Soc. 32, 44544457 (2011).

36. Xin, H., Lin, L.-X., Wu, J.-H. \& Yan, B. Hydrothermal synthesis and multi-color photoluminescence of GdVO4: $\mathrm{Ln} 3+(\mathrm{Ln}=\mathrm{Sm}$, Dy, Er) sub-micrometer phosphors. $J$. Mater. Sci. Mater. Electron. 22, 1330-1334 (2011).

37. Kalai Selvan, R., Gedanken, A., Anilkumar, P., Manikandan, G. \& Karunakaran, C. Synthesis and Characterization of Rare Earth Orthovanadate (RVO4; R = La, Ce, Nd, Sm, Eu \&amp; Gd) Nanorods/Nanocrystals/Nanospindles by a Facile Sonochemical Method and 
Their Catalytic Properties. J. Clust. Sci. 20, 291-305 (2009).

38. Gavrilović, T. V., Jovanović, D. J., Lojpur, V. \& Dramićanin, M. D. Multifunctional Eu3+and Er3+/Yb3+-doped GdVO4 nanoparticles synthesized by reverse micelle method. Sci. Rep. 4, 4209 (2015).

39. Yang, H. K. et al. Synthesis and Luminescence Properties Behavior of Eu 3+ -doped Nanocrystalline and Bulk GdVO 4 Phosphors by High-Energy Ball Milling and Solid State Reaction. J. Nanosci. Nanotechnol. 11, 474-478 (2011).

40. Nimmegeers, B., Cosaert, E., Carbonati, T., Meroni, D. \& Poelman, D. Synthesis and Characterization of GdVO4:Nd Near-Infrared Phosphors for Optical Time-Gated In Vivo Imaging. Materials (Basel). 13, 3564 (2020).

41. Cionti, C., Della Pina, C., Meroni, D., Falletta, E. \& Ardizzone, S. Triply green polyaniline: UV irradiation-induced synthesis of a highly porous PANI/TiO 2 composite and its application in dye removal. Chem. Commun. 54, 10702-10705 (2018).

42. Mielenz, K. D., Velapoldiand, R. \& Mavrodineanu, R. Standardization in Spectrophotometry and Luminescence Measurements: Proceedings of a Workshop Seminar Held at the National Bureau of Standards, Gaithersburg, Maryland, November. (1976).

43. Jovanović, D. J. et al. Up-conversion luminescence of GdVO4:Nd3+/Er3+ and GdVO4:Nd3+/Ho3+ phosphors under $808 \mathrm{~nm}$ excitation. Opt. Mater. (Amst). 82, 1-6 (2018).

44. Meroni, D., Gasparini, C., Di Michele, A., Ardizzone, S. \& Bianchi, C. L. Ultrasoundassisted synthesis of $\mathrm{ZnO}$ photocatalysts for gas phase pollutant remediation: Role of the synthetic parameters and of promotion with WO3. Ultrason. Sonochem. 66, 105119 (2020).

45. Mahapatra, S., Madras, G. \& Guru Row, T. N. Synthesis, Characterization and Photocatalytic Activity of Lanthanide (Ce, Pr and Nd) Orthovanadates. Ind. Eng. Chem. Res. 46, 1013-1017 (2007).

46. Rimoldi, L. et al. Impregnation versus Bulk Synthesis: How the Synthetic Route Affects the Photocatalytic Efficiency of Nb/Ta:N Codoped TiO2 Nanomaterials. J. Phys. Chem. C 119, 24104-24115 (2015).

47. Klimkevicius, V., Janulevicius, M., Babiceva, A., Drabavicius, A. \& Katelnikovas, A. Effect of Cationic Brush-Type Copolymers on the Colloidal Stability of GdPO 4 Particles with Different Morphologies in Biological Aqueous Media. Langmuir 36, 7533-7544 (2020).

48. Mohan, J. C., Praveen, G., Chennazhi, K. P., Jayakumar, R. \& Nair, S. V. Functionalised gold nanoparticles for selective induction of in vitro apoptosis among human cancer cell lines. J. Exp. Nanosci. 8, 32-45 (2013).

49. Yang, L., Li, L., Zhao, M. \& Li, G. Size-induced variations in bulk/surface structures and 
their impact on photoluminescence properties of GdVO4:Eu3+ nanoparticles. Phys. Chem. Chem. Phys. 14, 9956 (2012).

50. Surca, A. K., Dražić, G. \& Mihelčič, M. Low-temperature V-oxide film for a flexible electrochromic device: Comparison of its electrochromic, IR and Raman properties to those of a crystalline V2O5 film. Sol. Energy Mater. Sol. Cells 196, 185-199 (2019).

51. Soharab, M. et al. Spectroscopic properties and Judd-Ofelt analysis of Nd doped GdVO4 single crystals grown by OFZ method. Opt. Mater. (Amst). 92, 379-385 (2019).

52. Gu, L. et al. In vivo time-gated fluorescence imaging with biodegradable luminescent porous silicon nanoparticles. Nat. Commun. 4, 2326 (2013).

53. Ogawa, T. et al. Efficient laser performance of Nd:GdVO4 crystals grown by the floating zone method. in Advanced Solid-State Photonics 26 (OSA, 2003).

doi:10.1364/ASSP.2003.26

54. Romano, F. et al. Water-soluble silicon nanocrystals as NIR luminescent probes for timegated biomedical imaging. Nanoscale 12, 7921-7926 (2020).

55. Liu, X. et al. Tumor-Targeted Multimodal Optical Imaging with Versatile Cadmium-Free Quantum Dots. Adv. Funct. Mater. 26, 267-276 (2016).

56. Pons, T. et al. In Vivo Imaging of Single Tumor Cells in Fast-Flowing Bloodstream Using Near-Infrared Quantum Dots and Time-Gated Imaging. ACS Nano 13, 3125-3131 (2019).

57. Ortgies, D. H. et al. Lifetime-Encoded Infrared-Emitting Nanoparticles for in Vivo Multiplexed Imaging. ACS Nano 12, 4362-4368 (2018). 